Doi 2260

\title{
CORRIGENDA
}

\section{Avoiding premature licensing}

Mark Kessel and Sam Hall

Nature Reviews Drug Discovery 5, 985-986 (2006); doi: 10.1038/nrd2203

In the legend for Figure 1 (page 986), the definitions to 'Announced value' and 'total value' are incorrectly described.

'Announced value' in fact represents the overall 'Biodollars' announced publicly and the 'total value' is the company's estimate

of the overall value of all deals, including those for which there was no announced value. 